\title{
RFID Tags to Aid Detection of Buried Unexploded Ordnance
}

\author{
Keith Shubert and Richard Davis \\ Battelle Memorial Institute \\ United States
}

\section{Introduction}

Detecting buried unexploded ordnance (UXO) at military firing ranges and elsewhere is very difficult and expensive. To enable the military to conduct cost-effective training and research missions in the future, with increased safety for personnel and property without negative environmental impact, significant advances in detection and identification of buried UXO must be pursued and implemented.

This chapter presents the results of analytical and experimental efforts that demonstrated the viability of using munition-mounted radio frequency identification (RFID) tags as buried ordnance detection and identification aids. RFID tagging of ordnance can provide a high probability of detection and a near-zero false alarm rate. The tag provides discrimination between UXO and clutter items, a capability that is critical to reducing the cost of UXO remediation. This work was pursued because state-of-the-art passive RFID tags can potentially provide information on the munitions' locations while maintaining compatibility with operational deployment. To ensure success, however, detection range below the ground had to be investigated quantitatively.

The analysis was performed in the context of a UXO searching process that included the movement of the transmit and receive coils in pre-defined lanes across a large land parcel. The term "overlap" refers to the amount of overlap in two adjacent search lanes, i.e., the portion of area retraced by the coil. This planned movement of the interrogation system's transmit and receive coils will reduce the impact of isolated nulls or minima within the field patterns at both the tag and receive coil locations.

The operational concept calls for fastening tags to the exterior of candidate ordnance item as part of the manufacturing process. During the detection segment of the UXO remediation process, the UXO interrogation module provides energy to the tag by emitting a large magnetic field. The munition-mounted tag responds by emitting a low-level digital signal that is sensed by receivers on the UXO interrogation module. This research focused on lowfrequency, passive (non-battery) RFID tags, a choice made early in the investigation. Extensive modeling of the RFID tag on the metal munition was performed to aid in understanding the tag mounting parameters. The critical mounting concern is the required separation between the tag and the metal of the munition because the customer required detection of munitions buried as deep as one meter. For safety reasons, the transmitting coil and RFID tag must be shown not to induce a large electric field near the munition because too large a field might cause the munition to detonate. 


\section{Background}

Various methods for detecting buried unexploded ordnance at military firing ranges have been investigated (GAO, 2004), (Halman, et al., 1998). These techniques involve scattering energy off the munition and resolving the modified signal. Unfortunately, achieving consistent results using these methods has proved to be difficult and expensive. The techniques have also resulted in an unacceptable number of false-positive indications that will significantly increase the cost of remediation because of the expense of excavating nonUXO items. Using RFID tags, false alarms can occur only when a munition explodes and the RFID tag survives. This event is thought to have such a low likelihood of occurring that a statistical investigation of this scenario was not performed.

The method examined here differs from previous methods in that detection of the munition is based on the energy received from a transmitting RFID tag affixed to the munition, as depicted in Figure 1. The detection signal does not result from energy scattered by the munition itself.

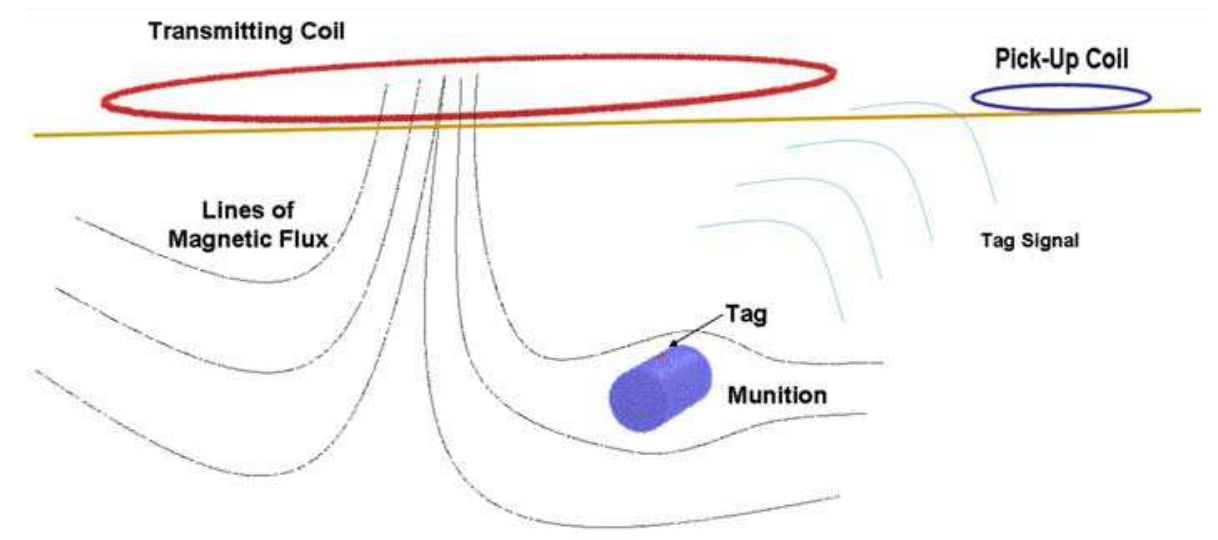

Fig. 1. Depiction of the tagged munition technique.

The ordnance detection system comprises an interrogation module and the RFID tag. The above-ground interrogation module, used to search for tags, generates a large magnetic field. The tag harvests the transmitted energy to power its integrated circuit and replies with a digital signal. The interrogation module's receive coil senses the digital signal transmitted by the tag, then reports detection and any embedded digital data, which might include information such as the munition type, serial number, and date of manufacture. One meter deep detection of UXO was deemed necessary to demonstrate feasibility.

After determining which passive RFID tags offer the highest probability of success in this application, additional electromagnetic and mechanical constraints were analyzed. Electromagnetic considerations involved characterization of the ability to energize and receive the reply from a passive RFID tag one meter from the excitation coil, the effect of the presence of the munition's metal on tag functionality, and the danger of the high-energy transmitting coil setting off buried munitions. Mechanical considerations involved finding ways to mount the RFID tags on existing metal munitions that allow RFID tag survival and functionality, subject to the extreme accelerations associated with munitions and ordnance. 


\section{System design}

\subsection{RFID tag choice}

According to Faraday's and Lenz' Law, the eddy currents induced on a conductive munition will tend to oppose the magnetic field perpendicular to its surface. This opposition becomes greater nearer the munition's surface. Therefore, magnetic fields very near the munition will tend to be parallel to its surface. This condition suggests choosing a solenoidal-shaped tag mounted with its core parallel to the surface, allowing the most efficient harvesting of energy from the interrogation module's magnetic field.

One candidate solenoidal tag was the Texas Instruments (TI) Tiris RFID tags. The decision was made to exploit these tags because of their operating frequency near $130 \mathrm{kHz}$ and their reliance on the magnetic field, both of which were favorable for maximum ground penetration of the transmitted energy. Figure 2 shows the Texas Instruments' Tiris solenoid tags employed during this study on the left and the in the larger "pancake" geometry on the right. The solenoid tags comprise a copper coil wrapped around a ferrite core, other circuit elements, and a digitally-based integrated circuit that functions as a receiver, transmitter, and processor with 64 bits of user-written data. The tags and readers employ frequency-shift keying (FSK) between $123 \mathrm{kHz}$ and $134 \mathrm{kHz}$ to transmit the tag's data to the reader.

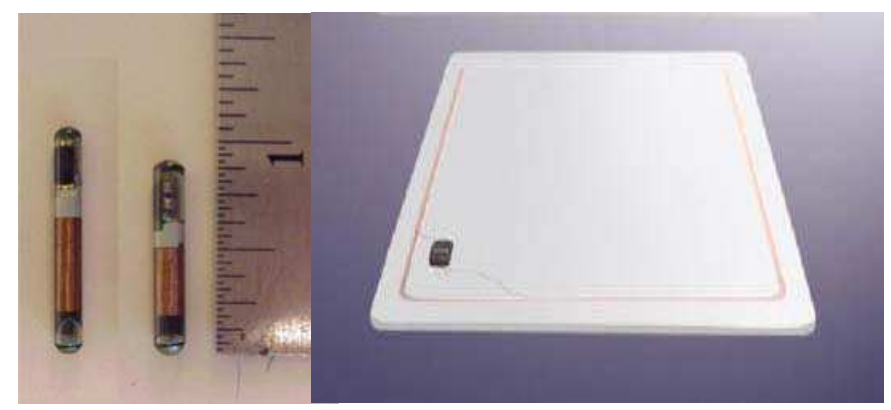

Fig. 2. Two examples of the low-frequency passive tags. The photo on the left shows the solenoid tag design; the coil wire is wrapped around a small permeable core. The photo on the right shows the "pancake" coil design used in non-contact facility access applications. The small black objects in the figures are the integrated circuits.

The RFID system choice was influenced by the Tiris tag's response mechanism being more favorable in this application. The Tiris tags do not reply until after the interrogation module's transmitter has turned off, which allows detection of the weak tag signal in a quieter spectral environment. Alternative RFID systems have tags that reply during reader interrogation, generally at half the reader's transmitted frequency. This simultaneous transmit and receive process in non-Tiris systems forces their readers' detection systems to detect very small signals in the presence of very large signals. Although this detection can be accomplished, it requires very precise relative positioning of the transmitting and receiving coils to minimize the magnitude of the transmitted signal cross-coupled into the receiving coil. It was anticipated that maintaining the relative positions of the coils precisely would be very difficult while traversing artillery and bomb ranges. The required positioning accuracy is much less for the Tiris system because the transmitter is turned off while the receiver is enabled. 


\subsection{The transmit coil}

The passive Tiris tags used in this study were intended to function with a separation between the reader and the tag of about one meter under certain conditions. Because budgetary constraints required that no changes be made to the RFID tags themselves, the interrogation module would have to be modified and made larger to achieve the required separation of one meter in the presence of the metal munition. The basic magnetic field equations were examined to determine the possible ways to increase the field levels at longer ranges. The magnetic field emitted in the $z$-direction, $B z$, by a circular loop of radius $a$ in the $x-y$ plane at a point located a distance $r$ from the origin can be expressed in $\mu \mathrm{W} / \mathrm{m}^{2}$ as

$$
B_{z}=\frac{\mu_{0} I N a^{2}}{2\left(a^{2}+r^{2}\right)^{\frac{3}{2}}}
$$

This relationship simplifies when the point $r$ is located far from the coil as

$$
B_{z}=\frac{\mu_{0} I N a^{2}}{2}\left(\frac{1}{r^{3}}\right) \quad \text { for } \quad r>>a
$$

Equation (1) holds in regions near the coil, where the reduction as a function of $r$ is slightly less than $1 / r^{3}$. The magnetic field increases linearly with the area of the coil, with the current, and with the number of turns. For a given value of $r$ (one meter) and a given value of area (radius of one-half meter to keep the interrogating module from being too large), it appears that one can arbitrarily increase $B$ by either increasing the number of turns, $N$, or the current, $I$. Practical considerations will limit the amount of current that can be employed but it still appears that arbitrary increases in the number of turns will yield increasingly large values of the magnetic field. Unfortunately, increasing the number of turns increases the inductance of the coil. The value of the inductance can be seen in equations (3) and (4) to increase as the square of the number of turns. The voltage across the coil is proportional to the inductance as seen in equation (5). This relationship implies that the voltage is proportional to $N$ squared. Thus, for a given coil current, an arbitrary increase in $N$ can quickly drive the voltage across the coil to impractical values. An optimum combination of $N$ and I must be determined given the amount of current the batteries and drive circuit can provide and the maximum tolerable voltage across the coil.

$$
\begin{gathered}
L=\frac{0.31(a N)^{2}}{6 a+9 h+10 b} \\
L=\frac{0.31 a N^{2}}{6} \quad \text { for } a>>h, b \\
V=L \frac{d I}{d t}
\end{gathered}
$$

The final interrogation coil design included a diameter of one meter and Litz wire with 270 strands of 38 American wire gauge (AWG) magnet wire. The coil design employed 10 turns of Litz wire $(N=10)$ and approximately 16 amps-rms, for a magnetomotive force of 160 ampere-turns-rms. These design values were confirmed to be effective through 
laboratory experimentation and electromagnetic modeling of the interrogation system and munition-mounted tag. No analytical optimization was attempted.

The laboratory investigation provided insight into potential detection ranges but it did not answer the basic physics question of whether or not the tag mounted on or near a metallic munition could function as needed.

\subsection{Modeling approach}

Knowledge of the magnetic field's behavior is essential in understanding the overall system designs required to transmit energy from the above-ground interrogator to the tag and from the tag back to the above-ground receiver. Quantities of interest, such as signal strength, can be calculated from these fields.

In the absence of the munition, the tag system's general behavior is well known. However, the tag's necessarily close proximity to the metallic munition will affect its operation. These passive tags are activated when there is sufficient magnetic energy linking its circuit's pickup coil. Getting the requisite level of magnetic field to the tag for activation will be impeded by the munition. Once activated, the tags generate a transmitted field that is altered by the presence of the metal munition also.

Furthermore, the operational circuitry of the tag requires a specific quality factor $(Q)$ to operate (Shubert, 2007, 2008). The $Q$-value depends on the inductance and resistance of the tag's pick-up coil. These values will be distorted in the presence of the munition. If the $Q$ value for the tag is too low, the tag cannot operate. There are of course other electromagnetic factors interfering with the tag's operation, but proximity to the munition is primary.

Figure 1 shows the basic geometry of the system as it was modeled. A transmitting coil sends out a signal. If a tagged munition is within its range, the tag is activated and sends out its own signal. The tag's output signal is picked-up by a receiving coil.

For purposes of the modeling investigation, this basic system was decoupled into two separate cases; (1) transmitting a signal to the tag and (2) receiving a signal from the tag. These two cases are independent because the amplitude of the signal received by the munition-mounted tag and the amplitude of the field transmitted by the tag are not related. In both cases, the electric field was examined for Hazards of EM Radiation to Ordnance (HERO) issues (Davis et al., 2006). The changes in Q-value of a tag were also examined separately.

\subsection{Magnetic modeling}

The models consisted of two- and three-dimensional finite element models. For this task, the commercially available finite element software package, Vector Fields, was used. Battelle has successfully employed this type of modeling in other studies. Those results were validated in both laboratory and field experiments.

Because the practical range of frequencies that will be used are on the order of a $100 \mathrm{kHz}$ and because the system antennas are in the near field, the models were solved using the low-frequency Maxwell's equations. For the two-dimensional models, equation (6) was solved subject to appropriate boundary conditions. Here, $A$ is the magnetic vector potential, $J_{s}$ is the source current density, $\sigma$ is the conductivity, and $\mu$ is the magnetic permeability.

$$
\nabla \times\left(\frac{1}{\mu} \nabla \times A\right)=J_{S}-\sigma \frac{\partial A}{\partial t}
$$


The curl of the vector potential, $A$, yields the magnetic field, $B$ and its negative partial time derivative yields the electric field, $E$. These two-dimensional models were used to provide quick qualitative insights into the basic behavior of this system.

Although more time consuming, the three-dimensional models provided more useful quantitative information. Equation (6) was used in the conductive regions and regions near conductive materials. In all other regions, the magnetic scalar potential shown in equation (7) was solved. Here, $\Phi_{m}$ is the magnetic scalar potential. The solutions are matched at the interfaces and are subject to appropriate boundary conditions. The gradient of the magnetic scalar potential, $\Phi_{m}$, yields the magnetic field, $B$.

$$
\nabla^{2} \Phi_{m}=0
$$

Rather than employ an open-boundary technique, the mesh was extended to a reasonable distance, a minimum of five times the distance between the munition and the transmit coil, with a scalar potential boundary condition applied to the outer surface. This method ensures that truncation has an insignificant effect on the region of interest. Although requiring larger model sizes than the open-boundary technique, past experience indicates that it provides a more accurate solution. The effect of truncation was estimated by calculating the tangential component of the field on an open boundary with a derivative boundary condition. Here, one-half the field observed on such a boundary is being reflected back from the exterior. Combining this value with knowledge of the probable decay in the exterior space gave an order of magnitude for the effect of the truncation on the regions of interest. The effect of truncation was less than one part in a hundred.

In general, the two-dimensional models consisted of about 100,000 elements and had an error relative to element size less than 0.25 percent. The three-dimensional models consisted of about 800,000 elements and had an error relative to element size less than 1.0 percent.

Figure 3 shows an example of the basic three-dimensional models used for these calculations including the cylindrical munition, a solenoid tag mounted on the munition, and the transmit coil. The soil and air are not shown, but their interface is located along the transmit coil's plane.

For calculating the fields from the transmitting coils and tags, a uniform and constant source current of 1 amp-turn was defined. The individual turns of transmitting coil wires need not be modeled, provided the individual wire size is small compared to the coil diameter and wavelength. Note that the source input is defined in terms of a constant current and not voltage. The relationship between voltage and current input depends on the circuitry used to drive the coils and is not important in modeling the fields.

Practically, the magnetic field levels generated will be such that the magnetic permeability of all likely materials is constant. Therefore, the electromagnetic field results, upon which all quantities of interest are calculated, will be linear with the number of amp-turns defined. The calculated results can then be scaled for any strength transmitting coil or tag.

Once field levels are determined, signals to and from the tags can be calculated using equation (8). Here $S$ is the signal strength, $\mathrm{N}$ is the number of turns in the receiving coil, $B$ is the magnetic field linking the receiving coil, and $a$ is the area of the coil. If a sinusoidal waveform is assumed, the right hand side of equation (8) is obtained. Here $u_{\text {eff }}$ is the effective permeability of a receiving coil's permeable core, $\omega$ is the angular frequency, and $B_{o}$ is the calculated magnetic field linking the circuit in the absence of core permeability. 


$$
\mathrm{S}=\mathrm{N} \int_{\text {Coil }} \frac{\mathrm{d} \overrightarrow{\mathrm{B}}}{\mathrm{dt}} \cdot \mathrm{d} \overrightarrow{\mathrm{a}}=\mathrm{N} \mu_{\text {eff }} \mathrm{B}_{\mathrm{o}} \omega \mathrm{a}
$$

The Texas Instruments tags specify a minimum $Q$ to respond to an interrogating signal. Models of the tags near the munitions were used to calculate the changes in the $Q$-value due to their proximity to the metallic munition (Skilling, 1957). $Q$ can be defined in several ways but two definitions stand out in this application. It is useful to define it as proportional to the ratio of the inductance to the resistance of the tag's receiving coil. It can also be defined as the ratio of the resonance frequency to the bandwidth, which is a measure of the relative narrowness of the resonance curve.
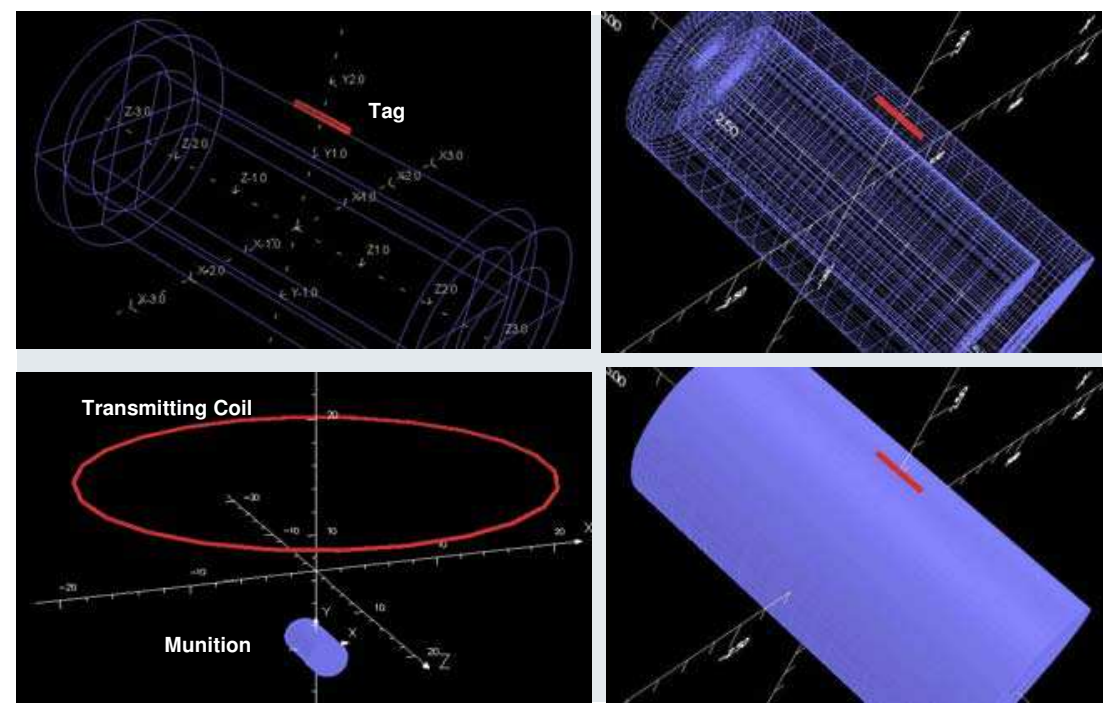

Fig. 3. The basic models for the munition, solenoid tag, and transmitting coil. The bottom left figure shows the transmitting coil and munition. Here the coil is centered directly over the munition and the ground is not shown. The other figures show the geometry (top-left and bottom-right) and mesh (top-right) for the munition model. The tag is also shown centered on the top surface of the model.

The resistance, $R_{c}$, of the tag in free space is known a priori. Using the value of the inductance, $\mathrm{L}$, and resistance, $\mathrm{R}$, caused by the nearby munition, the $Q$-value is defined as

$$
\mathrm{Q}=\omega \mathrm{L} /\left(\mathrm{R}+\mathrm{R}_{\mathrm{c}}\right)
$$

The value of $Q$ is dimensionless. The $\mathrm{L}$ and $\mathrm{R}$ values can be calculated from the electromagnetic fields. The value of $\mathrm{R}$ is related to the power loss of the tag caused by induced eddy current density, $J$, in the munition. If $I$ is the constant source current, the value of $R$ is given by

$$
\mathrm{R}=\frac{1}{|I|^{2}} \int_{\text {volume }} \frac{J^{2}}{\sigma} d V
$$


The value of $L$ is related to the magnetic energy created by the tag for a constant source current. If $B$ is the magnetic field as a function of space, the value of $L$ is given by

$$
L=\frac{1}{\mu|I|^{2}} \int_{\text {volume }} B^{2} d V
$$

The integrals are over all space containing conductive and/or permeable material.

The following parameters were examined to provide insight into the system with the intent of proving feasibility and optimizing the system. These parameters were studied for all three situations modeled using both two- and three-dimensional models. The parameters being varied included:

- Above-ground transmitter and receive coil positions relative to the munition

- Soil type and conductivity (dry dirt to salt water)

- Effect of nearby metallic or permeable materials

- Munition depth

- Munition orientation

- Munition shape

- Munition material

- Tag type (solenoid or pancake)

- Tag position on munition

- Tag orientation

- Tag mounting options such as tag offset from and "grooves" on the munition

- Frequency of operation (both transmitter and tag)

For each situation, a baseline model was defined. These parameters were varied relative to this baseline model to determine their effects.

\subsubsection{Models for transmitting the signal from the tag}

The fields at the munition surface near the above-ground receiving coil are critical to understanding behavior. The flux from the tag linking the receiving coil's windings will determine whether the tag's output signal can be detected.

The same basic munition geometry defined in the signal-to-the-tag case was used. The effects of the transmit coil were ignored. A specific tag was modeled as being fastened to the munition. Its location was variable. Both types of tags were modeled for baseline. Baseline was a tag centered on the top of the munition with the same offset.

A receive coil does not need to be specifically modeled. The signal received from the tag by a receive coil depends only on the magnetic field linking the receiver's coil windings. This value can be calculated independently using equation (8) once the model is solved.

\subsubsection{Models for estimating effects on Q-values}

Examination of the tags' changes in $Q$-value due to the proximity of metallic munition was done using two- and three-dimensional modeling. The model's field output was calibrated to commercial tags. Effects of various mounting techniques including separation (lift-off) from the metallic surface were examined. The electromagnetic fields solved for these cases were used to calculate the $Q$-value through the inductance and resistance using equations (9) through (11). 


\subsection{Magnetic modeling}

\subsubsection{Signal to the tags}

Modeling did provide insight into behavior of the fields near the munition generated by the transmitting coil.

As expected, the field amplitudes dropped significantly near the munition's conductive surface. The decrease in field and resulting signal to the tag can be as much as two orders of magnitude in going from a lift-off of $2.5 \mathrm{~mm}(0.1 \mathrm{inch})$ to the munition surface. In general, a 6.3-mm ( 0.25 -inch) gap increases the field by a factor 2.5 over a $2.5-\mathrm{mm}$ separation. The exact change depends on the munition material. As expected, lower conductivity in the metal ordnance object results in lower eddy current amplitudes and therefore lower loss in field level at the munition. Also, higher permeability of the material tended to increase the field near the munition. Nonetheless, the proximity of the tag to the munition will decrease the magnetic field near the tag. Therefore, the separation between the metallic surface and the tag is critically important.

In order to accommodate this required separation, the idea of grooves into which the tags would be placed was considered. Modeling indicated that groove shape has minimal influence on the field coupled into the tag. The important parameters were groove depth and groove length and width. Separation requirements were identical. The length and width of the groove should be such that there is about $5 \mathrm{~mm}(0.2 \mathrm{inch})$ of clearance between the tag's coil and the sides of the groove. This requirement holds for both tag types. The effect of the composition of the material potting the tag in the groove was also investigated. Nonconducting, permeable material will aid in coupling the interrogation signal into the tag.

Near the munition, the magnetic field lines tend to be parallel to the conductive surface. For a surface lift-off of $2.5 \mathrm{~mm}$, the magnetic field parallel to the munition surface can be as much as five times larger than the magnetic field perpendicular to the surface. This fact indicates that a tag with solenoid geometry will receive a much higher input signal than a tag with the pancake geometry.

Figure 4 shows the magnetic vector equipotential lines from a transmitting coil near the munition as it passes by. The magnetic field is parallel to these lines. Here, the metallic munition is oriented vertically and the coil passes over its centerline. Regardless of the coil position, the field lines tend to be parallel to the munition.

This behavior is a consequence of the electromagnetic boundary condition associated with conductive surfaces and strongly suggests that the pancake coil tag would not be feasible for this application because it requires magnetic fields perpendicular to the surface of the munition to be activated. It would be helpful to orient two solenoid tags axially and circumferentially on the munitions with circular cross-section. However, because of practical constraints, the solenoid tags can only be oriented axially on the munition. Therefore, the transmitting coil will have to produce sufficient axial field on the surface of the munition for this system to be feasible. The other parameters examined focused on this geometry.

The angular orientation of the ordnance item (vertical to horizontal) as the above-ground coils are moved, as they would be in a large ground-area survey, were examined. For a coil centered over the munition, the two extremes, vertical and horizontal, were modeled. In the first case the magnetic field on the surface of the munition is vertical. In the second case, the field is mostly circumferential, albeit larger in magnitude. 


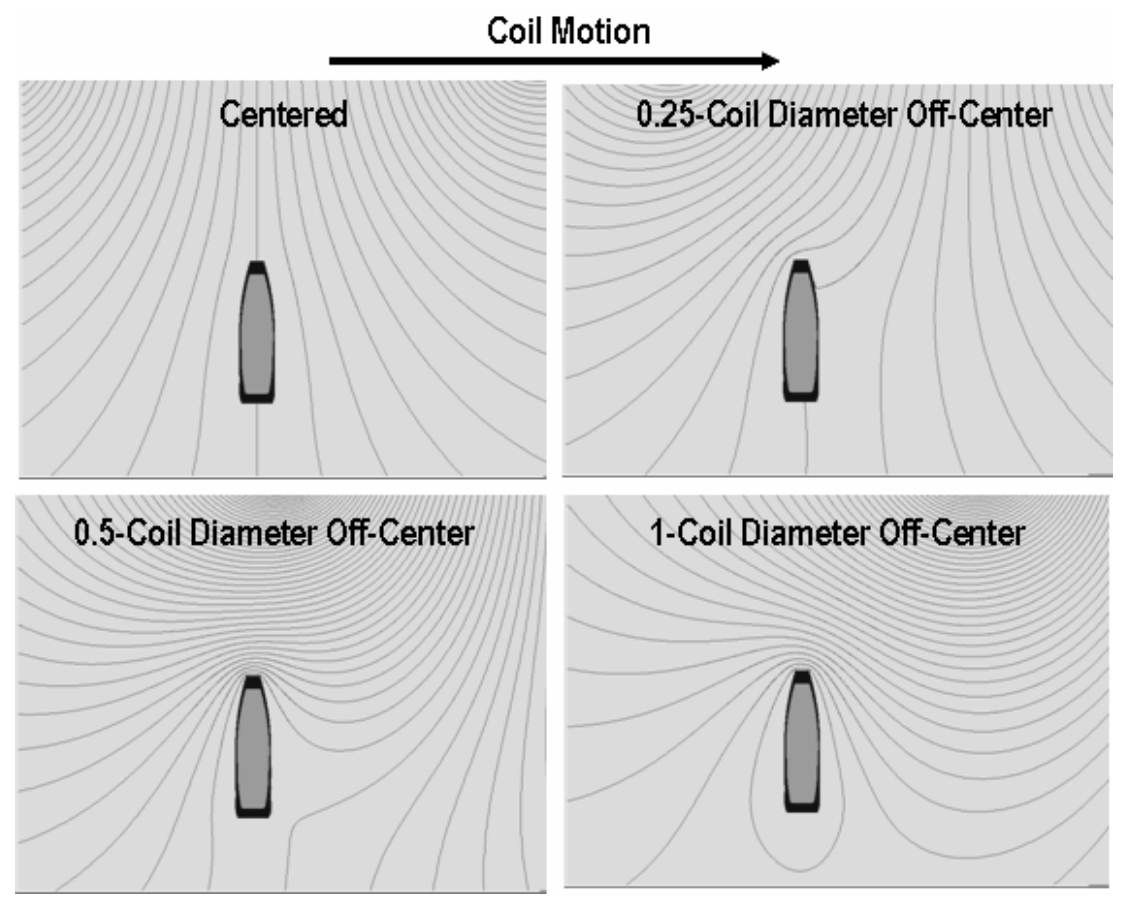

Fig. 4. Two-dimensional modeling results showing the magnetic vector equipotential lines from the transmitting coil as it passes directly over a vertically oriented munition. The magnetic field is tangential to these equipotential lines. The coil is not shown, but it is located one-half meter above the munition. The center of the coil is designated in the figures.

However, the orientation of the field on the munition will change as the transmitting coil passes over the ordnance item. Figure 5 shows an example. In all cases examined, there were at least some points along the coil motion that caused an axial field somewhere along the surface of the munition.

The relative signal received by an axially-oriented solenoid tag, for a munition whose axis is parallel to the surface, is shown in Figure 6. The two cases shown are for the coil passing parallel and perpendicular to the munition's axial axis.

In the first case, sufficient field can exist to activate the solenoid tag, although the transmitting coil will be off-center from the munition. In the second case, sufficient field can only exist if the tag is near the ends of the munition. In this case, a tag centered on the munition cannot receive any activating field and the munition will not be detected. Placement of the tag on the munition is important. It is preferable to place it near the edge of the munition.

Notice also that the peak activating fields for a tag occur at different transmitting coil positions depending on munition orientation and how the transmitting coil passes over the munition. For the above example, in the first case, the tag receives its peak activating field when the transmitting coil is roughly one-half diameter off-center from the munition; in the second case the peak activating field occurs when it is centered. 


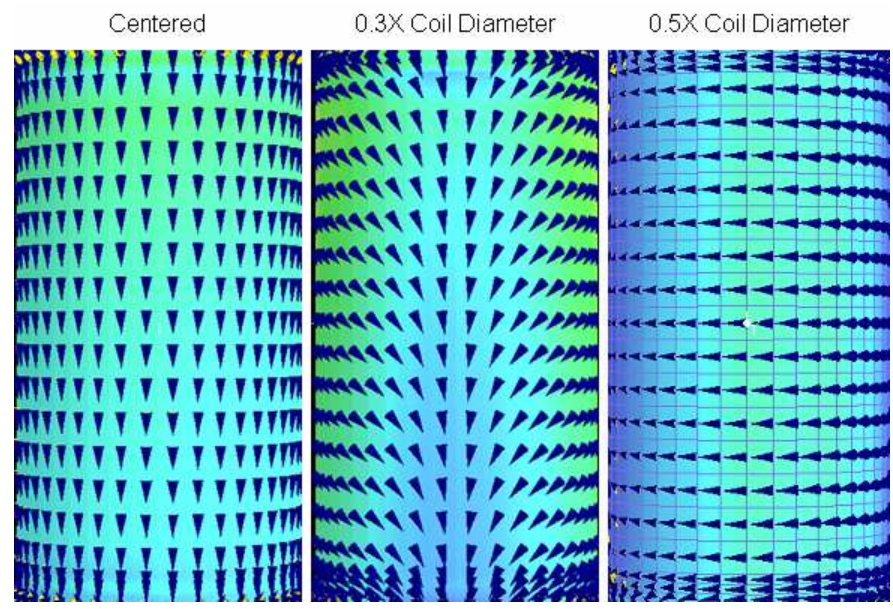

Fig. 5. The direction of the magnetic field on the surface of a vertically-oriented cylindrical munition with a one-meter diameter transmitting coil located 0.38 -meters above the munition, centered (left), 0.3-meter off-center (middle) and 0.5-meter off-center (right).
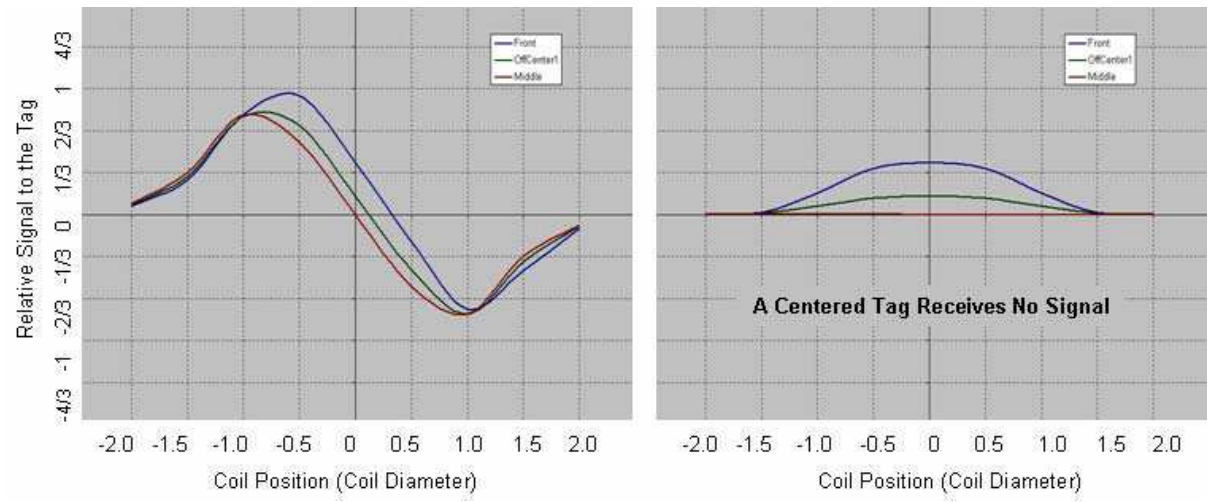

Fig. 6. Responses calculated as the interrogating coil moved past a horizontally-oriented munition. The left curves correspond to the transmitting coil moving parallel to the axis of the munition. The right curves correspond to the coil moving perpendicular to its axis.

For a given munition depth, the strength of the activating field varies only by about a factor of three depending on munition orientation, tag location, and where the transmitting coil passes. A vertical munition orientation provides the largest activating field. A horizontal orientation is more affected by coil motion and tag location.

For any given case, the magnitude of the field levels on the munition change only slightly as a function of the angular position. For a vertically oriented munition, the peak signal strength is independent of angular orientation. The worst case is for a horizontally oriented munition. In the horizontal case, the signal for a tag on the bottom of munition remains about one-half the magnitude at the top of the munition, which was encouraging because the munition's tag could be on the "underneath" side of the tag and still, be detectable. 
The basic behavior and orientation of the magnetic fields on the surface of the munitions do not change as a function of munition depth in the range of 0.3 to 1.5 times the transmitting coil diameter. The only change in field is the amplitude. In general, if a solenoid tag is mounted near the edge of the munition with at least a 6.3-mm lift-off, a signal should be detected by the tag for all cases up to a one-meter depth. If the solenoid tag is mounted at the center of the munition, the average maximum depth for tag activation reduces to about 0.5 meters; however, there are certain cases in which the tag will not receive any signal regardless of depth. For the pancake coil tag, the maximum depth is about 0.1 meters.

Figure 7 shows the amplitude of the peak axial field on the top surface of a horizontallyoriented munition. Assuming 100 amp-turns for a one-meter diameter transmitting coil and a solenoid tag, equation (8) suggests a field level of $8.5 \mathrm{pT}$ is required for tag activation. For this case, a maximum depth of more than 1 meter is possible. If the tags were located on the bottom of the munition, field levels for the horizontal case would be roughly halved, but still a one-meter deep tag can be activated. A vertically oriented munition would have a somewhat larger field.

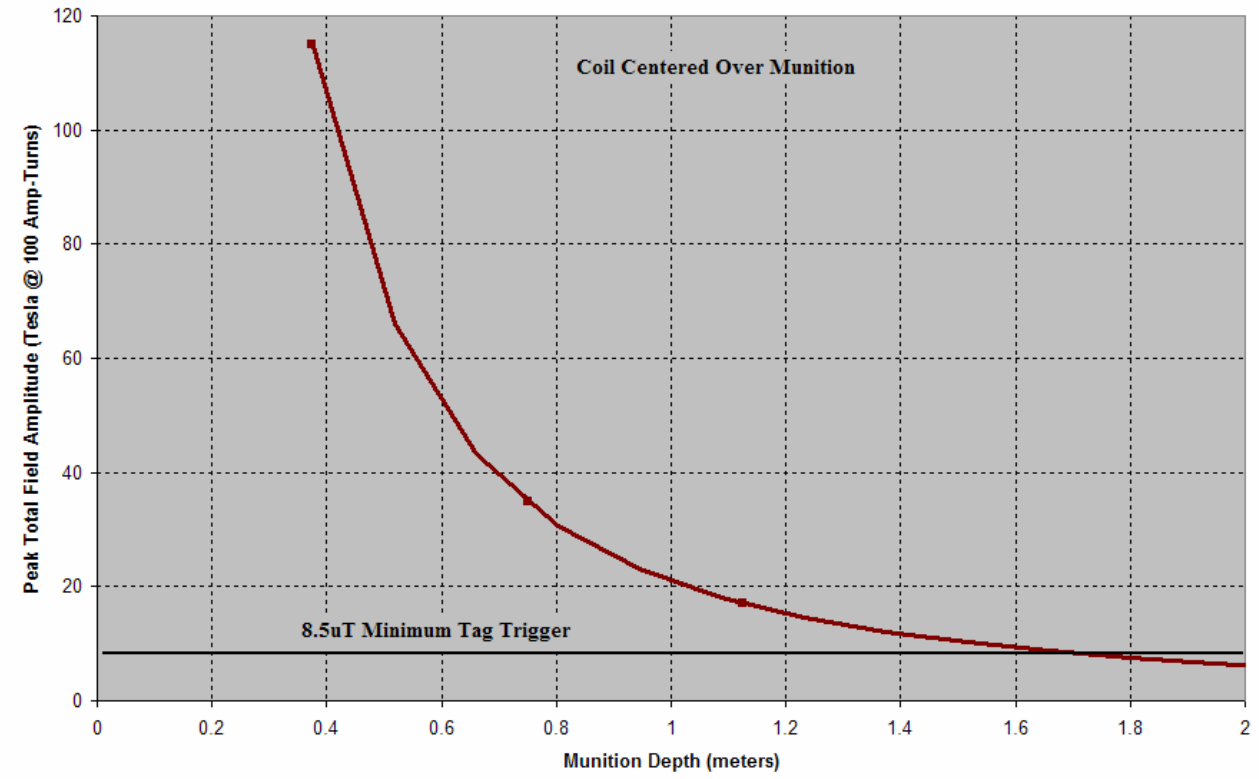

Fig. 7. The maximum field level on the surface of the munition as a function of munition depth. The coil is centered over munition.

Frequency of the transmitting coil's signal is also important. For practical considerations, the frequency range between 50 and $300 \mathrm{kHz}$ was examined because most commercial tags of interest fall into this range. In all cases, the field levels near the munition slightly decreased with increases in frequency. However, because the signals to the tags are linear with frequency, the signal increased monotonically. Commercially available tags are set to receive a fixed frequency. But, this information indicated that use of a higher frequency signal has advantages. 
Munition geometry was also studied. In general, there was no appreciable change in the behavior of the fields for the geometries analyzed. Smaller munitions allowed larger field levels near its surface. For a cone shaped munition, the field was slightly larger near the smaller diameter ends.

There are other factors that can alter the field on the surface of the munition. For example, the presence of other permeable or conductive material, such as the remnants of exploded ordnance items nearby, can shield the tag from the input signal. Modeling indicates that if larger pieces of ordnance items are at least 0.1 meter from the munition and each other, field levels near the munition will not be significantly affected. Otherwise, the conductive material will shield the munition's tag from the field.

Soil conductivity is generally of concern for higher frequency systems but it was examined in our study. The results indicate that if the frequencies are on the order of $150 \mathrm{kHz}$ and the munition depth is less than one coil diameter, soil conductivity does not affect the signal transmitted to the tag much. For a 0.38-meter deep munition and a frequency of $150 \mathrm{kHz}$, the difference between a dry soil and one saturated with salt water was a decrease in field amplitude of less than 20 percent.

If the tag mounting is optimized, modeling suggests that an overlap for the transmit coil of a half-coil diameter will be sufficient for ensuring that there is enough magnetic energy to actuate a tag at a one-meter depth.

The electric field at the munition produced by the transmitting coil was also calculated. Using these models, the current design of our one-meter diameter coil has been predicted to be about $0.5 \mathrm{~V} / \mathrm{m}$ at $100 \mathrm{kHz}$ immediately below the coil, which lies well below the HERO safety level. The HERO curves specify the maximum safe level at $100 \mathrm{kHz}$ to be between 10 and $40 \mathrm{~V} / \mathrm{m}$ (rms), depending on the sensitivity of the munition.

\subsubsection{Signal from the tags}

Once a tag has been activated, in generates its own output signal that is picked up at the surface using a receive coil. The magnetic field generated from both the pancake and solenoid tag geometries were examined. Figure 8 shows the magnetic vector equipotential lines from both tag types, in air and in a groove on the munition. The magnetic field is parallel to these lines and larger when the lines are closer together. The presence of the conductive munition reduces the field output of the pancake coil tag significantly while slightly boosting the output from the solenoid tag. The pancake coil tag generates a field normal to the surface of the munition that is reduced because of the electromagnetic boundary conditions. The effect on the solenoid is somewhat reversed, the metallic munition repels the magnetic field, which increases the signal transmitted by the tag boosting the effective signal output by as much as 20 percent.

Just as with receiving the signal from a transmitting coil, the solenoid tag performs better in outputting a signal. For these reasons, the solenoid geometry was the focus of the rest of this study. Because of practical constraints, these tags must be axially oriented on the munition so only axially oriented tag results are presented here.

Unlike inputting a signal to the tag, the effect of munition materials examined had little effect on solenoid tag output.

Soil conductivity has minimal effects on the tag's output signal as with the tags input signal. Again, the tag location on the ordnance item was found to be important with a tag position closer to the end of the munition increasing signal at the pick-up coil a maximum of 10 percent, depending on the specific case. 


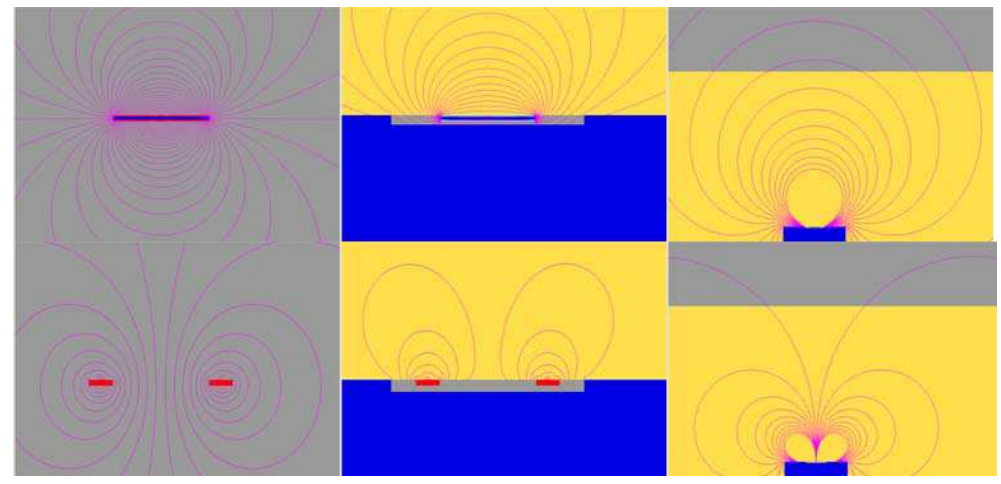

Fig. 8. Magnetic vector equipotential lines from the transmission of the solenoid (top row) and the pancake coil (bottom row) tags. The left column shows the results for the tags in air. The middle column shows the results for the tags in a grooved munition in dirt. These two columns are the same scale. The pancake coil tag's signal output is severely damped compared to the solenoid tag's signal because of the munition's presence. The right column shows the same results as the center column, but on a larger scale to see the impact near the surface.

When the tag is embedded in a groove, a wider and deeper groove is important but the shape of the groove is less important. Lift-off is not as much of a factor in getting signal to the surface as it was in getting signal to the tag. Here, a lift-off of only $2.5 \mathrm{~mm}$ is sufficient. A lift-off of $6.3 \mathrm{~mm}$ was required to receive the signal. The permeable filler placed in a groove to help increase input signal to the tag has little effect on tag output.

As in the case of the signal to the RFID tag, the tag's transmitted signal frequency was influential. As stated previously, the frequency range between 50 and $300 \mathrm{kHz}$ was examined during this work to correspond with commercial tag availability. While the magnetic field amplitude at the ground's surface slightly decreased as frequency increased, the signal in the above-ground receive coil increased monotonically with frequency.

The length of the solenoid tag was also examined. The longer tags produced a larger amplitude field at the ground surface. The commercial tags examined varied from 10 to $40 \mathrm{~mm}$ in axial length.

Figure 9 shows the peak amplitude of the three components of the magnetic field generated in a plane 0.38 meters above an off-centered, axially-oriented, solenoid tag. The columns show the axial, normal, and circumferential components, respectively. Two horizontal and one vertical munition orientations are shown. The red color is a positive and the blue color is a negative.

The basic field distributions depend only on munition orientation. The field amplitude decreases in amplitude as munition depth increases and as the tag angular location approaches the far side (bottom) of the munition.

These field distributions have significant consequences for the signal received by and the design of the receive coil. Only the field linking the receive coil's windings will be detected. For a point-coil receiver, the magnetic field amplitude is linearly proportional to the signal. However, because the coil will have a finite diameter, the average field level linking that coil area will determine the signal.

In practice, it will be easier to position the above-ground receive coil oriented parallel to the surface, so approach will focus on this case. In this situation, the normal component of flux induces the signal. 
Generally, a larger coil diameter will capture more flux and produce a larger signal for detection. However, the unique features in the tag's surface field distribution indicate that a coil diameter less than one meter should be sufficient given the munition sizes and depths of interest. Larger coil diameters would add little benefit.

Referring to Figure 9, the normal flux distributions are shown in the center column. The following arguments can be applied to any component of the flux detected by a pick-up coil. For a vertically oriented munition, the normal field distribution from the tag at the surface is a monopole. However, for a munition oriented parallel to the surface, this field distribution is a dipole.

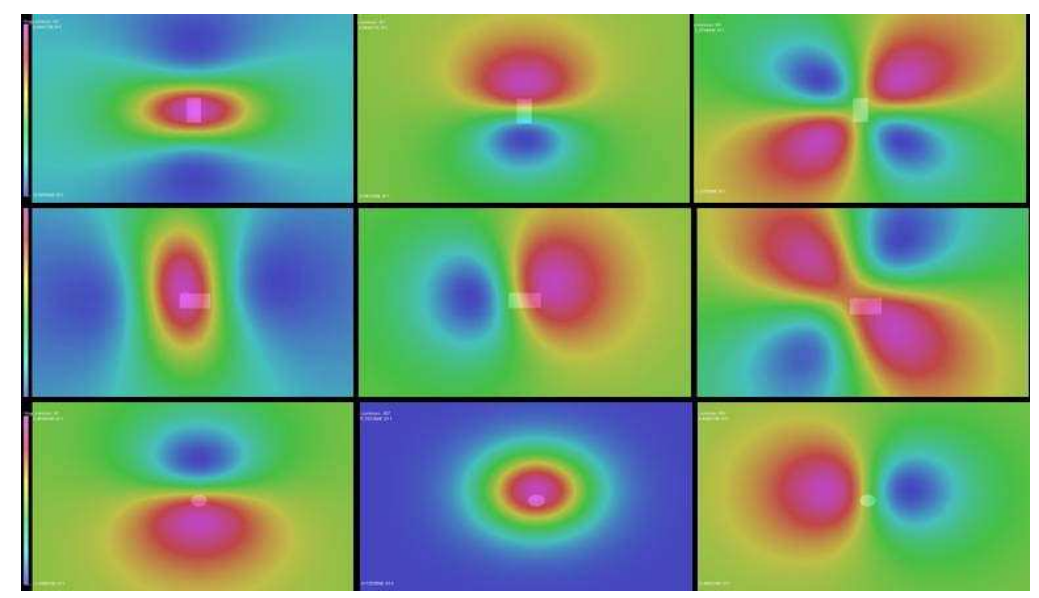

Fig. 9. The axial (left), normal (middle), and circumferential (right) components of the peak magnetic field transmitted by the solenoid tag at the surface. The top, middle, and bottom rows show the munition orientation parallel to the surface with centered tag on top of munition, same orientation rotated 90 degrees with the tag position off center on the side, and a vertically oriented munition with the tag centered, respectively. The munition is shown as a rectangle (parallel to surface) in the first two rows and a circle (vertical) in the last.

A problem arises with dipole distributions. Depending on the direction of the motion of the receive coil as it passes over the dipole field, the dipole field could tend to cancel itself as it links the coil windings. Therefore, it is very important that the receive coil have an overlap as it is scanning. This overlap will reduce the impact of isolated nulls inherent in the dipole field patterns because the system will be taking data continuously at nearby locations that are not within the null. Modeling suggests that the receive coils should have an overlap no less than one-half the coil diameter.

The models can be calibrated to any commercial tag. For example, the Texas Instruments' Tiris 32-mm long solenoid tag's specifications state that it has a field output between 80.5 and $102.5 \mathrm{Amps} /$ meter at $50 \mathrm{~mm}$ with an output frequency of $134 \mathrm{kHz}$. The calibration data are used to determine the maximum munition depth that can be detected realistically.

The field at the receive coil is dependent on the discussed parameters. It is also dependent on the receive coil's electronic design. Electronics associated with the receive coils can generally measure minimum signal strengths of about $5.0 \mu \mathrm{V}$. For practical purposes, 
assume the receiver has 20 turns, a coil area of $0.3 \mathrm{~m}^{2}$, and an effective core permeability of one. For this case, equation (8) suggests an average field strength of 6 picoTesla (pT) at the receiver is required for detection.

If the lower value for the tag output is used and the above receive coil is assumed, analysis indicates that a signal from the tag can be detected for a munition depth of least one meter, for all cases considered. The average field linking this receive coil as a function of munition depth is shown in Figure 10.

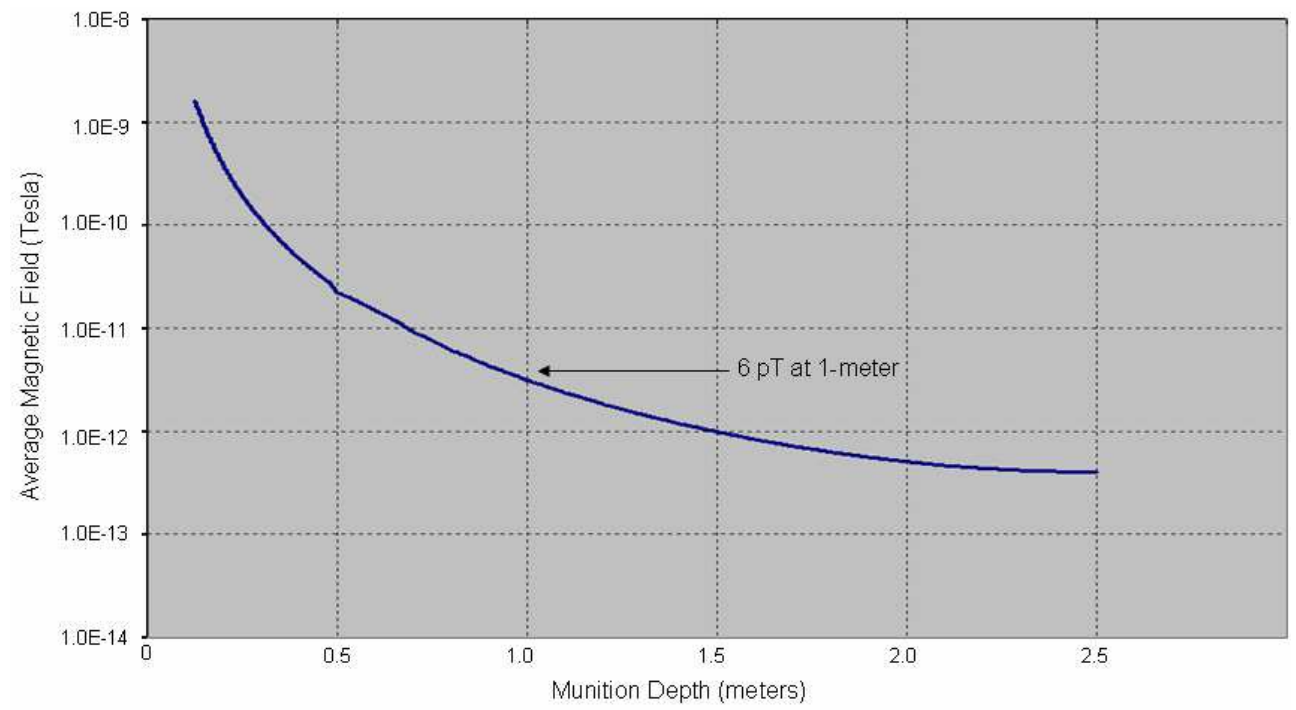

Fig. 10. The average field as a function munition depth. The munition is oriented parallel to the surface. For a typical receive coil, a field of $6 \mathrm{pT}$ is required.

The electric field produced by the tags at the munition was also calculated. The models indicate that the tags would produce electric field amplitudes less than $0.01 \mathrm{~V} / \mathrm{m}$ at $100 \mathrm{kHz}$ on the munition, far below the HERO safety levels.

\subsubsection{Q-value of the tags}

The Texas Instruments' tags specify a minimum quality factor above 60 to respond to an interrogating signal. In free space, the $Q$ of the transponder coils was found to be about 94 . The modeled results were calibrated with respect to these commercial tag values.

Modeling studies indicated that the metal of the munition casing had a significant impact on the $Q$ of the tag. As expected, the presence of the munition decreased the inductance and increased the resistance of the tag's circuit, thereby lowering the $Q$-value.

Figure 11 shows the induced eddy currents that change the resistive value of the circuit. Modeling indicates that in general a lift-off greater than $6 \mathrm{~mm}$ from the munition surface is required to keep the $Q$-value above 60 .

If the tag is placed in a groove, not only is this lift-off still required, but the tag should be separated from the walls of the groove by about $5 \mathrm{~mm}$. The nonconducting, permeable material used to aid in coupling the interrogation signal into the tag did not lower the $Q$-value. 


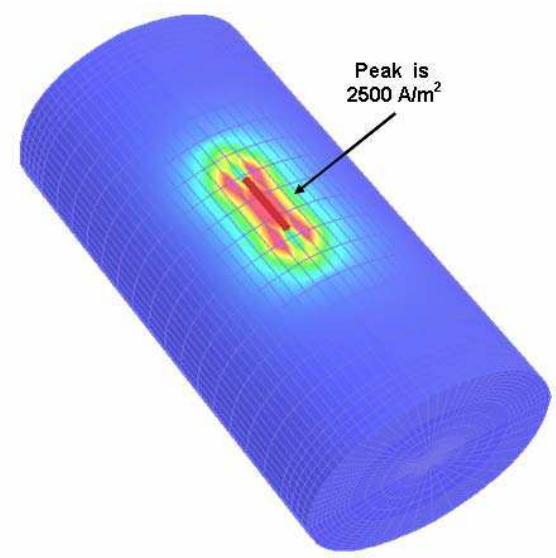

Fig. 11. The induced eddy currents from a solenoid tag on the surface of the munition. The peak current density for a typical commercial tag is about 2500 amps per square meter.

\subsection{Experimental verification}

Experimental efforts involved the design, fabrication, and tuning of the custom coil circuits. The tuning circuits for the high-voltage, one-meter diameter transmit coil and the corresponding receive coil were built.

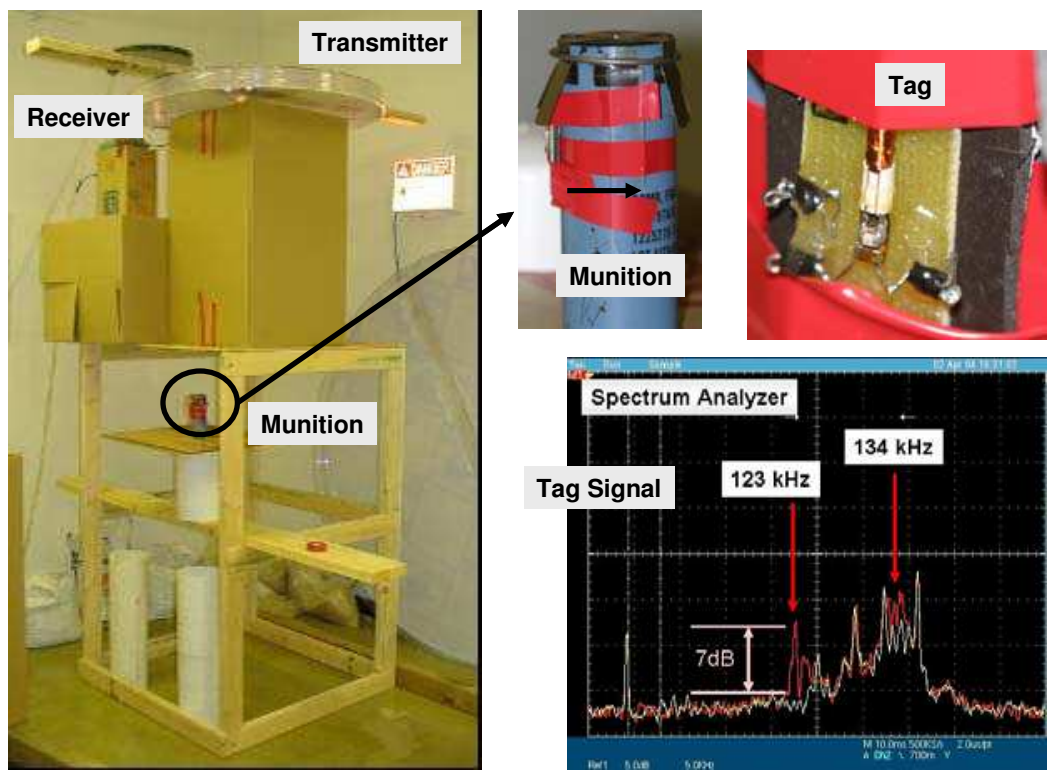

Fig. 12. The laboratory setup for obtaining experimental data. The left picture shows a onemeter diameter transmitting coil centered over a munition. The top right pictures show a close-up of this munition and the RFID tag. The lower right graphic is a spectrum analyzer screen shot displaying the two frequencies transmitted by the tag. 
$\mathrm{Lab}$ and field testing were conducted. The basic results of the modeling were verified, although not every parameter was examined in the lab and field. Tag proximity to the munition surface was important not only for receiving a signal but also maintaining a high $Q$-value. The separation distance of $6.3 \mathrm{~mm}(0.25 \mathrm{inch})$ for good tag performance was found to be adequate.

The solenoid tag and the one meter diameter coil in the laboratory using the set-up shown in Figure 12 were characterized. The solenoid Tiris tags from Texas Instruments have been used exclusively.

Experiments were performed to determine the distance from a transmitting coil that a tag could be activated by measuring the voltage level at the tag coil without a munition item. Ranges greater than 2 meters were observed. These experiments were repeated with the tag near a munition. Similar results were seen as long as the tag separation from the munition was sufficient.

Later experiments observed the field generated by the tag at the above-ground receiving coil. For optimized conditions, munition depths greater than one meter were detectable in the lab.

Tagged munitions were also detectable in the experimental field trials in dry clay soils. These findings also supported the modeling results.

\subsection{Modeling conclusions}

This modeling effort suggests that munition tagging, making use of current passive RFID tag technology, as a method to improve locating UXO and discriminating UXO from clutter is feasible.

In tagging the munition, a solenoid type tag was found to be preferable. The tag separation from the metallic munition surface is important for ensuring acceptable operation of the tag. A separation distance of $6.3 \mathrm{~mm}$ is required. If it is placed in a groove, separation of the tag from the groove walls should be about $5 \mathrm{~mm}$.

For practical reasons, the tag will be oriented axially on the munition and should be place near the ends of the munition so that the tag can receive a signal from the transmitting coil regardless of munition orientation. If centered on the munition, there are circumstances that would prevent a tag from receiving a signal. The tag can still receive and transmit sufficient signals regardless of its angular position on the munition, i.e., top or bottom.

The receive coil should be less than one meter in diameter. The transmit and receive coils should have an overlap of about one-half coil diameter. Soil conductivity did not present a problem.

For an optimized system, a detectable munition depth of a one meter is likely.

\section{Mechanical considerations}

The mechanical considerations were two-fold. First, the tag had to survive launch acceleration and impact. Second, the tag had to be mountable on existing munitions without significant modification to the munition.

Launch acceleration and velocity testing explored tag survivability potential. These tests were conducted at Battelle's West Jefferson, Ohio munitions testing facilities. A "soft catch" was employed using a combination of Styrofoam and duct tape to reduce deceleration forces. Tiris tags were removed from their glass containers, potted, and placed inside polypropylene cylinders that were inserted as shotgun shell loads. Tag survival was 
determined using a Tiris reader to monitor the tag's digital response. Initial results were encouraging. A single tag was fired 11 times; it survived the first 10 events. The results are listed in Table 1. The final firing at $67,000 \mathrm{~g}$ 's allowed the polypropylene slug to strike a steel plate, which did incapacitate the tag. Shot number 8 is highlighted in the table. The tag described in this line survived accelerations up to $43,000 \mathrm{~g}^{\prime} \mathrm{s}$ and a maximum velocity of 247 meters per second (809 feet per second). Informal discussions with ordnance experts indicated the highest acceleration/deceleration levels experienced by U.S. munitions were in the low 20,000 g's (anecdotal information did not indicate if this value was acceleration or deceleration). Further testing and analysis would be needed to ensure tag functionality meets acceleration/deceleration rates acceptable to the Government.

\begin{tabular}{|c|c|c|c|}
\hline $\begin{array}{c}\text { Shot } \\
\text { Number }\end{array}$ & $\begin{array}{c}\text { In-Bore } \\
\text { Acceleration (g's) }\end{array}$ & $\begin{array}{c}\text { Velocity } \\
\text { (feet/second) }\end{array}$ & $\begin{array}{c}\text { Tag } \\
\text { Response }\end{array}$ \\
\hline 1 & 10,000 & 340 & Good \\
\hline 2 & 27,000 & 277 & Good \\
\hline 3 & 33,000 & 681 & Good \\
\hline 4 & 12,750 & 375 & Good \\
\hline 5 & 26,900 & 575 & Good \\
\hline 6 & 10,800 & 381 & Good \\
\hline 7 & 15,850 & 438 & Good \\
\hline 8 & 43,100 & 809 & Good \\
\hline 9 & N/A & 882 & Good \\
\hline 10 & N/A & 475 & Good \\
\hline 11 & 67,500 & 1158 & Bad* \\
\hline \multicolumn{4}{|c|}{${ }^{*}$ Load struck metal plate behind Styrofoam } \\
\hline
\end{tabular}

Table 1. Summary of tag acceleration and velocity survivability testing

The initial research into the mounting feasibility and potential approaches was analytical; no physical testing was performed during this investigation. Five candidate munitions were considered for tagging. The candidates included munitions that were used at firing ranges and that stayed within one meter of the surface when they entered the ground and did not explode. These munitions were the BLU-97, MK-52 practice bomb, M720, M229, and $155 \mathrm{~mm}$ projectile. Because these munitions vary considerably in physical shape and size, no universal attachment method seemed realistic. However, mounting a tag to each munition type was deemed feasible with minor modifications to the munition. Distinct techniques were developed for each of these munitions. Figure 13 shows one potential approach for the MK-52 practice bomb.

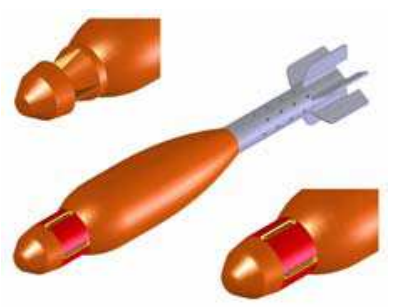

Fig. 13. One example of mechanically mounting a tag on a munition . 


\section{Evaluation and testing}

Experimental efforts involved the design, fabrication, and tuning of the custom coil circuits. The tuning circuits for the high-voltage, one-meter diameter transmit coil and the corresponding receive coils were built. The basic results of the modeling were verified, although not every parameter was examined in the lab and field.

\subsection{Lab evaluation}

Figure 12 shows the laboratory setup for characterizing the one-meter transmit coil and the Tiris tags. The Tiris tag was mounted on a BLU-97 munition $4.3 \mathrm{~mm}(0.17$ inch) from the steel body using a $2.8-\mathrm{mm}$ thick ferrite spacer and a $1.5-\mathrm{mm}$ thick circuit board spacer. The circuit board allowed probes to be attached for monitoring voltage levels. The frequencydomain view of the Tiris tag's response shows the frequency shift keying between $123 \mathrm{kHz}$ and $134 \mathrm{kHz}$.

Experiments were performed to determine the distance from a transmitting coil that a tag could be activated by measuring the voltage level at the tag coil. Ranges greater than two meters were observed in this configuration. These lab experiments were repeated with the tag mounted on an inert munition. Similar results were seen as long as the tag separation from the munition was sufficient. Later experiments observed the field generated by the tag at the above-ground receiving coil. For optimized conditions, munition/detection coil separations greater than one meter were achieved in the lab with the tag on a munition. Tagged munitions were also detectable at one meter in the experimental field trials in dry clay soils. These findings supported the modeling results.

As expected and predicted by the modeling, the tag proximity to the munition surface was important not only for receiving a signal but also maintaining a high $Q$-value so the tag could respond. In the lab setting with the BLU-97 munition, a separation distance of $4.3 \mathrm{~mm}$ ( 0.17 inch) produced acceptable results. This separation was less than the $6 \mathrm{~mm}$ minimum predicted by theory because a layer of ferrite $2.8 \mathrm{~mm}$ thick was placed between the tag and the ordnance item.

Even at separations greater than two meters, signal-to-noise ratios greater than $5 \mathrm{~dB}$ were observed when averaging was performed. Figure 12 shows a spectrum analyzer view of the FSK signal with a 7-dB signal-to-noise ratio. The data sets were taken with a digital oscilloscope that measures voltage waveforms as a function of time. The digital portion of the integrated circuit in the Tiris tag outputs a digital data stream of 64 bits that include synchronization information and information stored in its memory. This digital waveform then drives modulation circuitry that, in simple terms, transmits a tone at $123 \mathrm{kHz}$ to represent a logical "zero" and a tone at $134 \mathrm{kHz}$ to represent a logical "one."

This type of frequency-domain plot is useful for detecting the presence of a digital FSK signal but it is not useful for determining the information content of the digital signal. The ultimate detection system will demodulate the FSK signal and determine the underlying data. Such detail was not necessary for this proof-of-concept program. In a future effort, processing could be added to the detection circuitry that would perform the Fourier transform to convert the data to the frequency domain and allow signal processing that would identify low-level signals in the presence of noise and interference.

\subsection{Field testing}

The tagged-ordnance system was tested at the Aberdeen Test Center (ATC) located at Aberdeen Proving Ground. Nine tagged surrogates were buried with three untagged 
surrogates buried near the tagged surrogates to simulate the presence of buried clutter. ATC provided the location and depth information of the buried objects after their locations were predicted. The detection system shown in Figure 14 was used to transmit the tag activation signal and receive the tags' responses. The transmit coil is one meter in diameter and the receive coils are $30.5 \mathrm{~cm}$ (12 inches) in diameter.

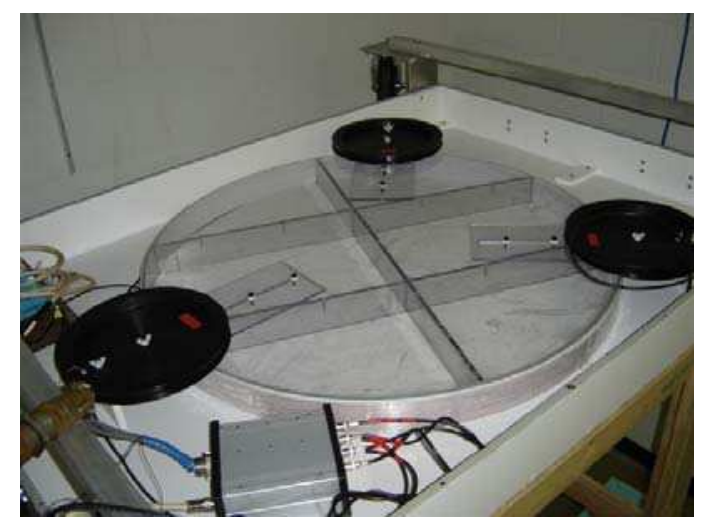

Fig. 14. The interior of the coil module showing the one-meter diameter transmit coil and the three separate receive coils.

The surrogates were solid steel cylinders that were $5 \mathrm{~cm}$ (2 inches) in diameter and $30.5 \mathrm{~cm}$ (12 inches) long. The nine surrogates had grooves milled into their surface into which tags were embedded. A photo of one of the tagged cylinders is shown in Figure 15. The tag had $5.65 \mathrm{~mm}(0.22 \mathrm{inch})$ of material between it and the surrogate munition including $2.85 \mathrm{~mm}$ of plastic next to the cylinder and $2.8 \mathrm{~mm}$ of ferrite next to the tag.

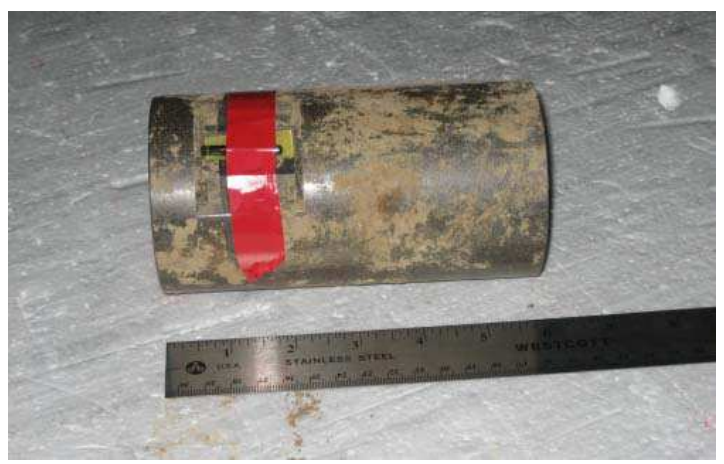

Fig. 15. Munition surrogate later buried at Aberdeen Test Center. The tag is evident below the red tape.

The nine targets were buried in a fairly small area because there was no need to test search rate at this point in the development cycle. ATC buried the objects in a four-meter by four-meter area.

The signals from the three receive coils were plotted on the oscilloscope after the scope performed Fourier transforms on the signals. Example frequency-domain plots are shown in 
Figure 16. The left and right vertical yellow cursors are positioned at 120 and $130 \mathrm{kHz}$, respectively. The left figure shows the signal from an untagged surrogate (essentially background noise). The figure on the right represents the background noise-plusinterference environment; signal strength is significantly higher at the two cursors. The tag is replying to the interrogating signal by switching between 123 and $134 \mathrm{kHz}$.
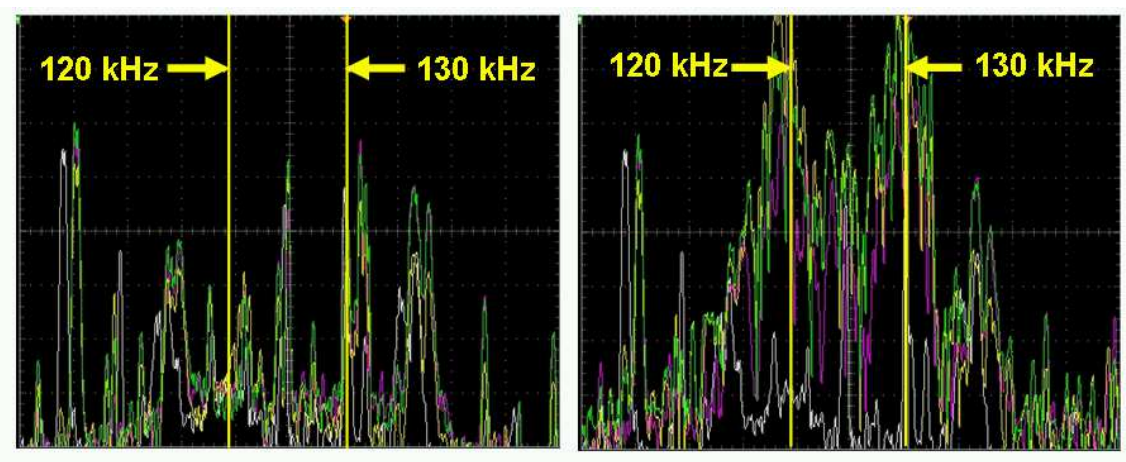

Fig. 16 (Left) Example data plot with no tagged surrogate present. (Right) Example data plot with coil module positioned above a tagged surrogate; signal strength is much higher at the two cursor positions.

The locations of the surrogates (tagged and non-tagged), Battelle's predicted positions, and data acquisition points are shown in the plot of Figure 11. The square markers indicate the positions of the tagged surrogates, the three triangles indicate where non-tagged surrogates were placed, the large circles represent Battelle's predicted positions of the tagged surrogates, and the diamonds indicate the position of the center of the transmit coil when data sets were recorded. The depth of each tagged surrogate is indicated with text. The predicted positions are indicated with large-diameter circles because of the large "footprint" of the coil module. The circle represents the uncertainty in the predicted position of the buried tag, an uncertainty similar to resolution cell size in an image. The actual resolution cell size was not determined experimentally, but modeling indicates it is a function of munition depth and orientation. A shallow munition might be detectable when the center of the coil module is one meter from the position of the buried tag but a one-meter-deep tag might only be detectable when the center of the coil is within $25.4 \mathrm{~cm}$ (10 inches) of a position directly over the tag.

Eight of the nine buried tagged surrogate positions were predicted. As stated previously, nine tagged surrogates were sent to ATC but the actual number buried was unknown at the time of the test. Figure 17 indicates the reasonable success in predicting the positions of the eight targets.

One tagged surrogate was missed completely by the predicting process. The missed surrogate, which was buried one meter below the surface, is shown in Figure 12 at position $(0.5,2.5)$. It was described by ATC personnel as "Tagged surrogate parallel to the surface with the tag down." The phrase "tag down" implies the tag's position on the surrogate is underneath the item, as far from the surface and the coil module as possible and in the untagged surrogate's shadow. The untagged surrogate was $15.2 \mathrm{~cm}$ (6 inches) above and parallel to the tagged surrogate. The figure shows that a data set was taken directly over the missed surrogate. After Battelle provided its predictions and ATC supplied the actual 
positions, the data set taken at $(0.5,2.5)$ was examined to see if the tag's signal existed in the data set. In fact, the tag's signal was very strong in the frequency-domain plot. It was concluded that the analysis and decision-making processes used for this test were too simplistic. An improved algorithm was needed.

Although this tagged surrogate's location was not predicted, it is encouraging that the tag's signal is very strong in the data even though it is at maximum depth at worst case position. Improved data presentation, analysis, and interpretation will allow similar tags to be correctly predicted in future assessment exercises.

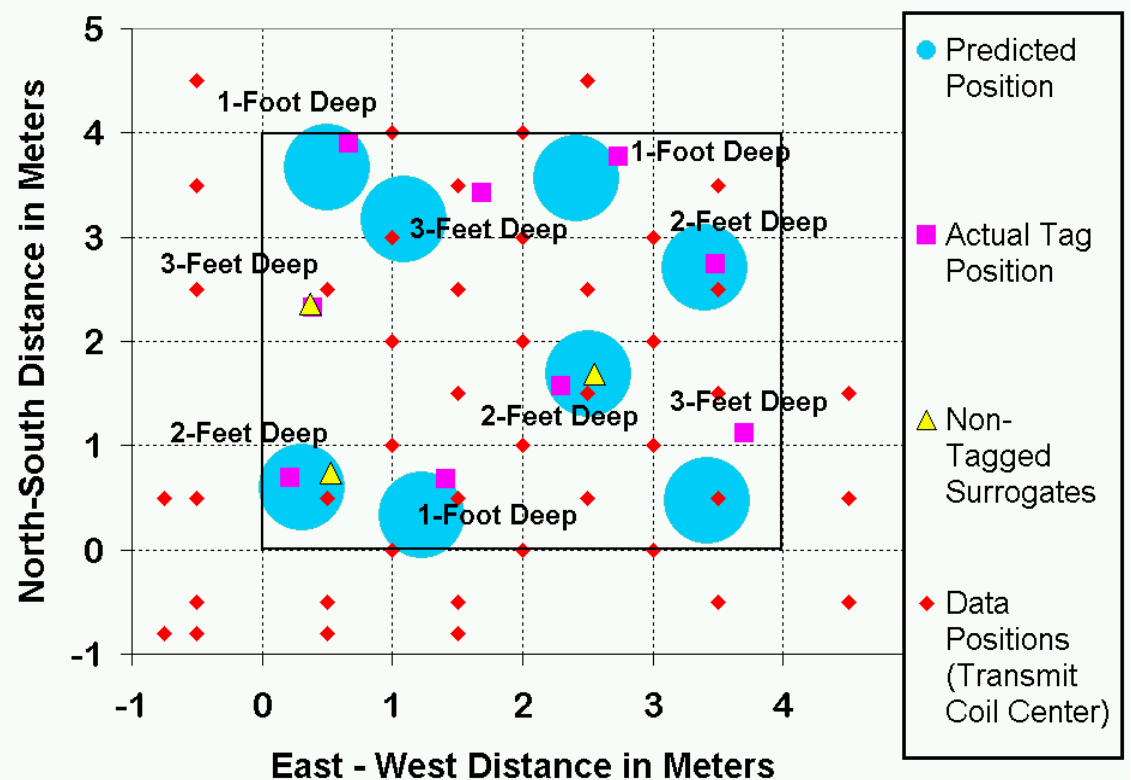

Fig. 17. Plan view of the test grid at Aberdeen Test Center showing the actual tagged munition positions, Battelle's predicted positions, and the positions where Battelle acquired data.

\section{Summary}

This study showed that the use of RFID tags to aid detection of UXO was feasible. Tags with solenoidal geometry, similar to the TI Tiris tag, are preferred for practical signal detection reasons. These tags show promise for being able to be mounted on munitions as well as surviving launch and impact. Analysis provided insight into the parameter variations necessary for optimizing the system, such as mounting the tags with some separation from the munition surface to allow sufficient magnetic energy into the tag and to allow the tag to operate properly. Field testing with the UXO interrogation system prototype was successful. Efforts incorporating further optimal design and detection algorithm adjustments remain.

\section{Acknowledgements}

This work was conducted for the Strategic Environmental Research and Development Program (SERDP) by Battelle Columbus Operations. 


\section{References}

Davis, R.; Shubert, K., Barnum, T. \& Balaban, B. (2006). Buried Ordnance Detection: Electromagnetic Modeling of Munition-Mounted Radio Frequency Identification Tags, IEEE Transactions on Magnetics, Vol. 42, No. 7, (Jul. 2006) pp. 1883-1891, ISSN 0018-9464

GAO Report (2004). DoD Operational Ranges; More Reliable Cleanup Cost Estimates and a Proactive Approach to Identifying Contamination Are Needed, General Accounting Office Report GAO-04-601

Halman, J.; Shubert, K. \& Ruck, G. (1998). SAR processing of ground-penetrating radar data for buried UXO detection: results from a surface-based system, IEEE Transactions on Antennas Propagation, Vol. 46, No. 7, (Jul. 1998), pp. 1023-1027, ISSN 0018-926X

Purello, M. (2002). New Maximum Allowable Environments (MAE) For HERO Unsafe Ordnance and HERO Susceptible Ordnance, Dahlgren Division, Naval Surface Warfare Center, Systems Electromagnetic Effects Branch (J52)

Shubert, K. ; Davis, R., Barnum, T., Balaban, B., Amdor, R., Sikorski, B., Peters, T., \& Griffin, J. (2007). Enhanced Electromagnetic Tagging For Embedded Tracking Of Munitions And Ordnance During Future Remediation Efforts, Final Technical Report on Project MM-1272 prepared for the Strategic Environmental Research and Development Program

Shubert, K.; Davis, R., Barnum, T. \& Balaban, B. (2008). RFID Tags to Aid Detection of Buried Unexploded Ordnance, IEEE Antennas and Propagation Magazine, Vol. 50, No. 5, (Oct. 2008), pp. 13-24, ISSN 1045-9243

Skilling, H (1957). Electrical Engineering Circuits, Second Edition, John Wiley and Sons, Inc., New York 


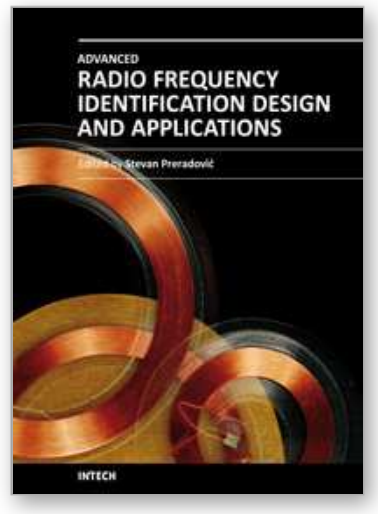

\author{
Advanced Radio Frequency Identification Design and Applications \\ Edited by Dr Stevan Preradovic
}

ISBN 978-953-307-168-8

Hard cover, 282 pages

Publisher InTech

Published online 22, March, 2011

Published in print edition March, 2011

Radio Frequency Identification (RFID) is a modern wireless data transmission and reception technique for applications including automatic identification, asset tracking and security surveillance. This book focuses on the advances in RFID tag antenna and ASIC design, novel chipless RFID tag design, security protocol enhancements along with some novel applications of RFID.

\title{
How to reference
}

In order to correctly reference this scholarly work, feel free to copy and paste the following:

Keith Shubert and Richard Davis (2011). RFID Tags to Aid Detection of Buried Unexploded Ordnance, Advanced Radio Frequency Identification Design and Applications, Dr Stevan Preradovic (Ed.), ISBN: 978-953307-168-8, InTech, Available from: http://www.intechopen.com/books/advanced-radio-frequency-identificationdesign-and-applications/rfid-tags-to-aid-detection-of-buried-unexploded-ordnance

\section{INTECH}

open science | open minds

\author{
InTech Europe \\ University Campus STeP Ri \\ Slavka Krautzeka 83/A \\ 51000 Rijeka, Croatia \\ Phone: +385 (51) 770447 \\ Fax: +385 (51) 686166 \\ www.intechopen.com
}

\author{
InTech China \\ Unit 405, Office Block, Hotel Equatorial Shanghai \\ No.65, Yan An Road (West), Shanghai, 200040, China \\ 中国上海市延安西路65号上海国际贵都大饭店办公楼 405 单元 \\ Phone: +86-21-62489820 \\ Fax: +86-21-62489821
}


(C) 2011 The Author(s). Licensee IntechOpen. This chapter is distributed under the terms of the Creative Commons Attribution-NonCommercialShareAlike-3.0 License, which permits use, distribution and reproduction for non-commercial purposes, provided the original is properly cited and derivative works building on this content are distributed under the same license. 